\title{
Conductivity and Photoconductivity of a p-type Organic Semiconductor under Ultra-Strong Coupling
}

\author{
Kalaivanan Nagarajan, 1 Jino George, $1 \$$ Anoop Thomas, 1 Eloise Devaux, 1 Thibault Chervy, 1 \\ Stefano Azzini, 1 Aziz Jouaiti, 2 Mir W. Hosseini,2 Anil Kumar, , Cyriaque Genet, 1 Nicola Bartolo, 4 \\ Cristiano Ciuti4 and Thomas W. Ebbesen.*
}

1University of Strasbourg, CNRS, ISIS \& icFRC, 67000 Strasbourg, France ${ }_{2}$ University of Strasbourg, CNRS, Laboratoire de Tectonique Moléculaire \& icFRC, Institut Le Bel, 67070 Strasbourg, France

3Indian Institute of Technology Bombay, Department of Chemistry, Powai, Mumbai 400076, India

4Université de Paris, Laboratoire Matériaux et Phénomènes Quantiques, CNRS, Paris 75013, France

KEYWORDS: Strong coupling, organic semiconductors, conductivity, photoconductivity, $p$ type

\begin{abstract}
During the last decade, it has been shown that light-matter strong coupling of materials can lead to modified and often improved properties which has stimulated considerable interest. While charge transport can be enhanced in n-type organic semiconductors by coupling the electronic transition and thereby splitting the conduction band into polaritonic states, it is not clear whether the same process can also influence carrier transport in the valence band of $p$-type semiconductors. Here we demonstrate for the first time that it is indeed possible to enhance both the conductivity and photoconductivity of a p-type semiconductor rr-P3HT that is ultra-strongly coupled to plasmonic modes. It is due to the hybrid light-matter character of the virtual polaritonic excitations affecting the linear-response of the material. Furthermore, in addition to being enhanced, the photoconductivity of rr-P3HT shows modified spectral response due to the formation of the hybrid polaritonic states. This illustrates the potential of engineering the vacuum electromagnetic environment to improve the opto-electronic properties of organic materials.
\end{abstract}


Light-matter strong coupling is a promising approach for controlling properties of matter.1-29 For instance, it has been shown that charge transport can be enhanced by an order of magnitude in the n-type perylene di-imide family of compounds and that the rate of energy transfer can be boosted, leading to nearly unit transfer efficiency.11-18 Furthermore, energy transfer can even be achieved over distances well beyond what is expected from Förster theory by entangling donor and acceptor to the same cavity mode.16-18 The modification of magneto-transport properties, with a reduction in the longitudinal resistance, has recently been predicted 30 and reported for ultra-strongly coupled 2D electron gas.31

The improved transport properties of organic materials are the result of the delocalised character of the electronic excited state in the collective strong coupling regime. However, the role of hybrid light-matter states on the transport characteristics of $p$-type semiconductors, where valence band holes are the majority carriers have not yet been studied, although it is also potentially important for organic electronics.32-38 Interestingly, based on the theory of intersubband cavity polaritons,38 one can predict that when excitons are coupled to a confined optical mode and one reaches the ultra-strong coupling regime, the ground state shifts while acquiring polaritonic character (cf. Supporting Information (SI)).

The fundamental question we explore here is whether this polaritonic character of the ground state and its excitations leads to enhanced conductivity in p-type semiconductors. For that purpose, we have studied a well-known p-type semiconductor, the regio-regular poly-(3hexylthiophene) (rr-P3HT) whose structure is shown in Figure 1a. Since photoconductivity plays a fundamental role in many technological applications such as photodetectors, electrostatic imaging and photovoltaics,39-45 we also explore whether the photoconductivity can also be improved in the ultrastrong coupling regime.

Strong light-matter coupling is achieved by placing a material with a well-defined transition in a resonant electromagnetic field such as a surface plasmon or a Fabry-Perot cavity. Under the right conditions, this strong interaction leads to the formation of two new hybrid light-matter states, known as polaritonic states $P+$ and $P$-, as illustrated in Figure 1 b.

\section{RESULTS AND DISCUSSION}


The conductivity and the photoconductivity of the rr-P3HT were studied on Ag nano-hole arrays (having hexagonal periodicity) between drain and source electrodes, using the same approach as in ref. 11. Ag was chosen for its capacity to sustain low-loss surface plasmon modes in the wavelength range of interest while still providing good injection capacity for the electrical measurements.11,33 As detailed in the Experimental section, the hexagonal hole arrays

(a)

(b)
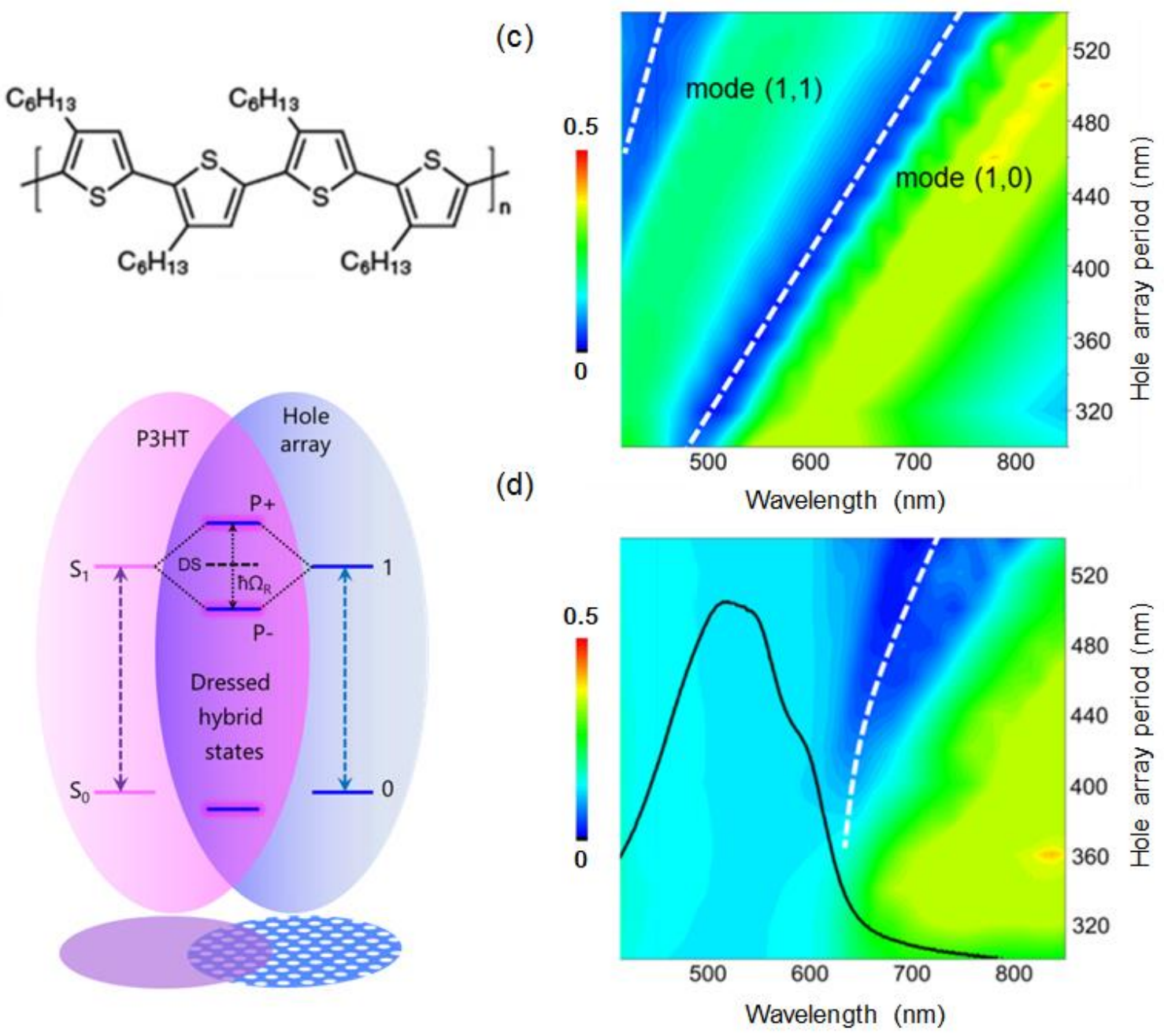

Figure 1. Strong coupling between surface plasmon and organic semiconductor: (a) Molecular structure of the p-type semiconductor rr-P3HT polymer. (b), Schematic representation of ultra-strong coupling between Ag hole array and rr-P3HT film. Contour image of transmission spectra of the hole arrays in the (c) absence and (d) presence of rrP3HT (black curve shows the molecular absorption) as a function of period ranging from $320 \mathrm{~nm}$ to $540 \mathrm{~nm}$ (dashed white lines are guidelines to eyes) (x-axis data is not shown below $415 \mathrm{~nm}$, the lower wavelength limit of our optical spectrophotometer detection). 
were milled with a Zeiss Auriga dual beam FIB/SEM in a $100 \mathrm{~nm}$ thick Ag film and the period P of the holes were varied (from $320 \mathrm{~nm}$ to $560 \mathrm{~nm}$ ) in such a way that the plasmonic resonances (Figure S1, SI) are in the same range as the first absorption bands of the semiconductor. The organic semiconductor rr-P3HT was prepared and purified as described in the literature.46 It was then dissolved in trichloroethylene by Soxhlet extraction method and spin-coated on the hole arrays, yielding ca. $85 \mathrm{~nm}$ thick films. The sample was then covered with a $200 \mathrm{~nm}$ PVA (poly vinyl alcohol) film. The strong coupling regime was verified by measuring their transmission spectra for different hole array periods $\mathrm{P}$ and comparing them with the bare hole arrays (i.e. without absorber) (Figure 1c and d). We observe a mode bending of the lower polaritonic branch $(P-)$ in the presence of organic semiconductor. $P+$ is in the UV region and cannot be detected with optical microscope with which the spectra were taken. The resulting Rabi splitting are estimated to be ca. $0.6 \mathrm{eV}$ for rr-P3HT corresponding to $27 \%$ of the transition energy as explained in the Supporting Information and in agreement with the theoretical analysis presented below. Such large Rabi-splitting indicates that the system is in the ultra-strong coupling regime where all the states of the system are modified with significant consequences as we will discuss further down.

The conductivity of the samples was then characterized using the setup schematically shown in Figure 2a. The I-V (current-voltage) curves of the various samples were then recorded as shown in Figure $2 \mathrm{~b}$ as a function of the hole period over a voltage range (-5 to $5 \mathrm{~V})$. From the measured I-V curve, current enhancement factor was calculated by comparing the current in the periodic hole array systems with that of a random hole array. Figure $2 \mathrm{c}$ plots the current enhancement factor at $\pm 5 \mathrm{~V}$ as a function of $2 \pi / \mathrm{P}$ for dark current of rr-P3HT coated hole arrays with respect to a random array. The current enhancement factor peaks when the absorption maximum of rr-P3HT crosses the $(1,0)$ and $(1,1)$ modes of the hexagonal plasmonic array (Figure 2d) and undergoes strong coupling. In other words, this occurs only when the plasmonic array is in resonance with the rr-P3HT absorption. The small differences, $3 \%$ and $8 \%$, between the crossings of the $(1,0)$ and $(1,1)$ modes with the rr-P3HT absorption (Fig. 2d) and the current peaks (Fig. 2c) is well within experimental error when considering the following. The SP modes position can only be calculated in the absence of the absorber. Obviously, the refractive index and therefore the SP modes will be modified by the presence of the absorber even in the absence 
of strong coupling. In addition, the rr-P3HT absorption is so broad that it overlaps with both modes simultaneously.

(a)

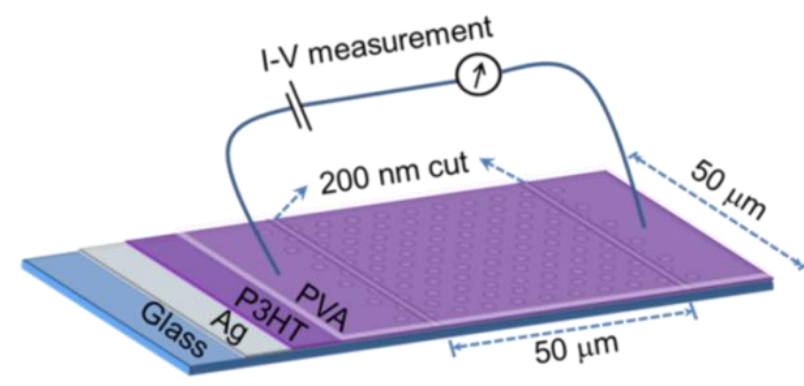

(b)

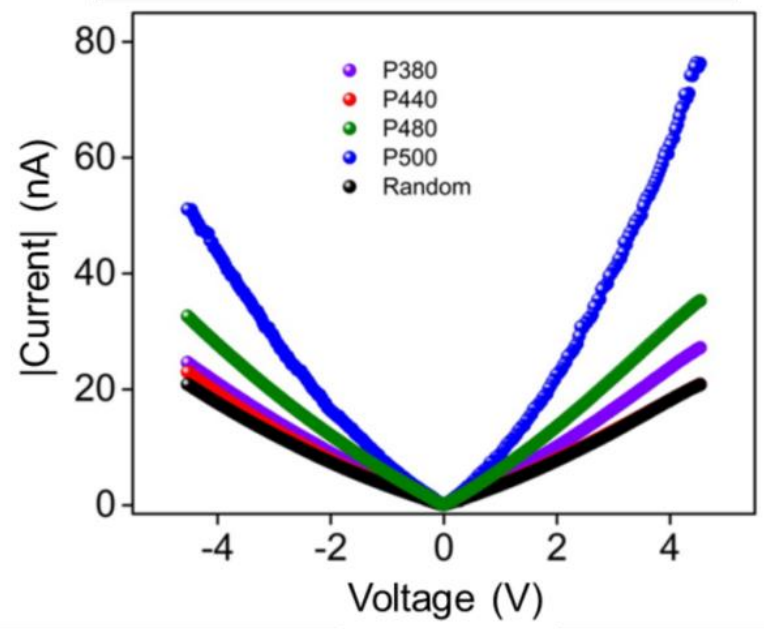

(c)
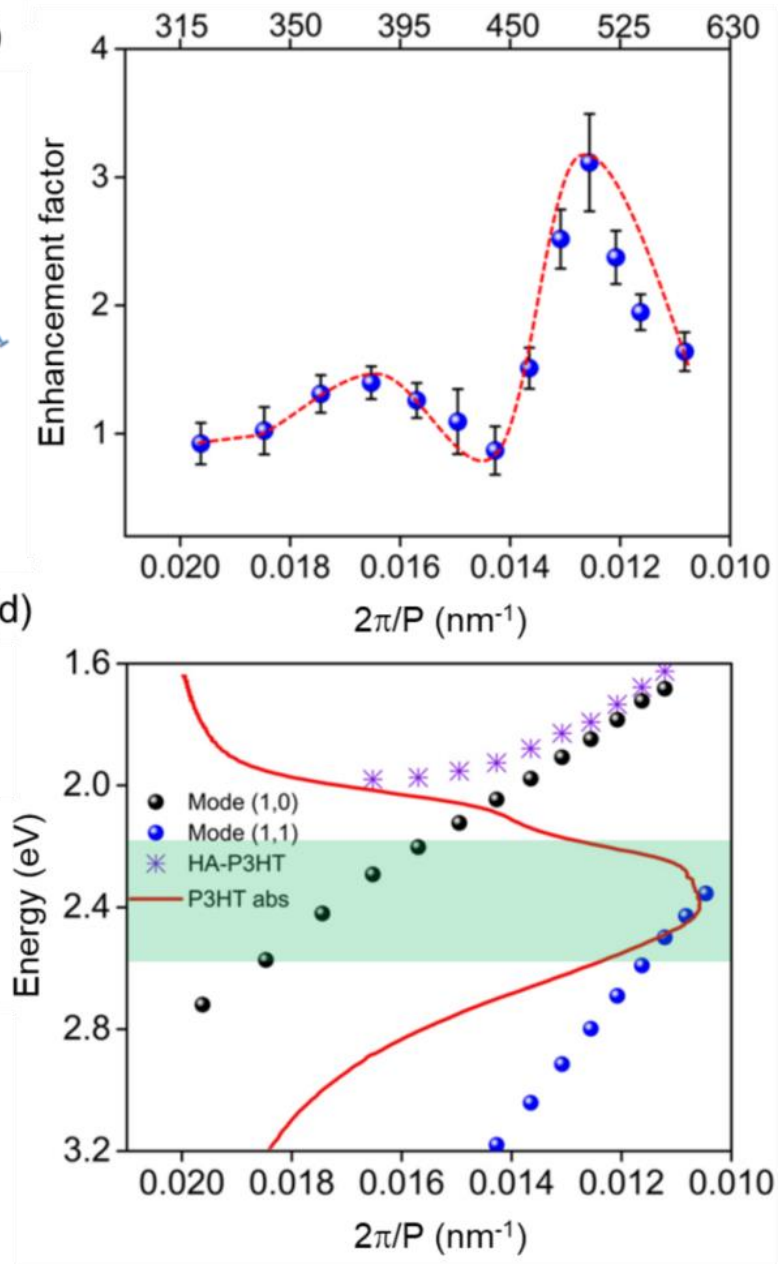

Figure 2. Current-Voltage measurement: (a) Schematic representation of the set-up used to perform conductivity measurement. (b) Current-voltage (I-V) curves of the rr-P3HT samples as a function of the period $P$ of the hole arrays. (c) Conductivity enhancement with respect to the in-plane momentum of hole arrays (top axis shows corresponding hole array period in $\mathrm{nm}$ ). Blue spheres show the average value of three different experiments and the error bars show the standard deviation. Red dotted line is guide to the eye. (d) The conductivity enhancement observed in c, correspond to the intersection of the rr-P3HT molecular absorbance (red line) and the surface plasmon resonance for the $(1,0$; black sphere) and (1,1; blue sphere) modes of hexagonal hole arrays in Ag; The violet stars show the lower polaritonic band. The broad absorption region of rr-P3HT is highlighted. 
When compared to the conductivity on a random hole array, rr-P3HT on resonance hole array $\left(\mathrm{P}=500 \mathrm{~nm} ; k_{\mathrm{x}}=0.0126 \mathrm{~nm}-1\right)$ exhibits an enhancement factor of $\sim 3$ for the dark current measurement. This enhancement is smaller than the maximum observed for a n-type perylene diimide semiconductor where it reached a factor of 10. As we demonstrated by comparing three different n-type semiconductors, the disorder in the material appears to have a large negative effect on the enhancement.15 So one possible explanation for lower enhancement in rr-P3HT, as compared to the best n-type PDI semiconductor we have tested, is the higher disorder.

A number of control experiments were carried out in order to exclude possible artifacts. The presence of oxygen also can modify the conductivity of rr-P3HT compounds so we checked that the results under nitrogen atmosphere for our coated samples were the same within experimental error. The metal substrate is floating and does not contribute to the conductivity due to the Schottky barrier at the semiconductor-metal interface. This was confirmed by depositing a $20 \mathrm{~nm}$ insulating $\mathrm{SiO}_{x}$ layer between the metal hole array and organic semiconductor which gave very similar results (Figure S2, SI). It was also verified that the observed boosts in current were not due to the cuts that are made in the hole arrays. As shown in the SEM image of the array after the cutting (Figure S3a, SI), the edge is smoothed by the FIB and finally random hole arrays, which have no pronounced plasmon mode, displays no enhancement (Figure S3b, SI).

This is the first example of enhanced conductivity for a p-type semiconductor under strong coupling which indicates that the charge carrying valence band is also affected by the presence of polaritonic states even in the absence of illumination. As mentioned earlier, the system is in the ultra-strong coupling regime under collective coupling conditions where counter-rotating terms and diamagnetic effects cannot be neglected. The effects of the light-matter coupling on the conductivity can be predicted via a many-body Kubo linear-response approach.30, 47 The transport properties of the material crucially depend on the hybrid light-matter nature of the ground state and of the virtual polaritonic excitations affecting the transport via a renormalization of the current response and scattering times. As detailed in the Supporting Information, we have calculated the conductivity mediated by virtual polariton excitations by 

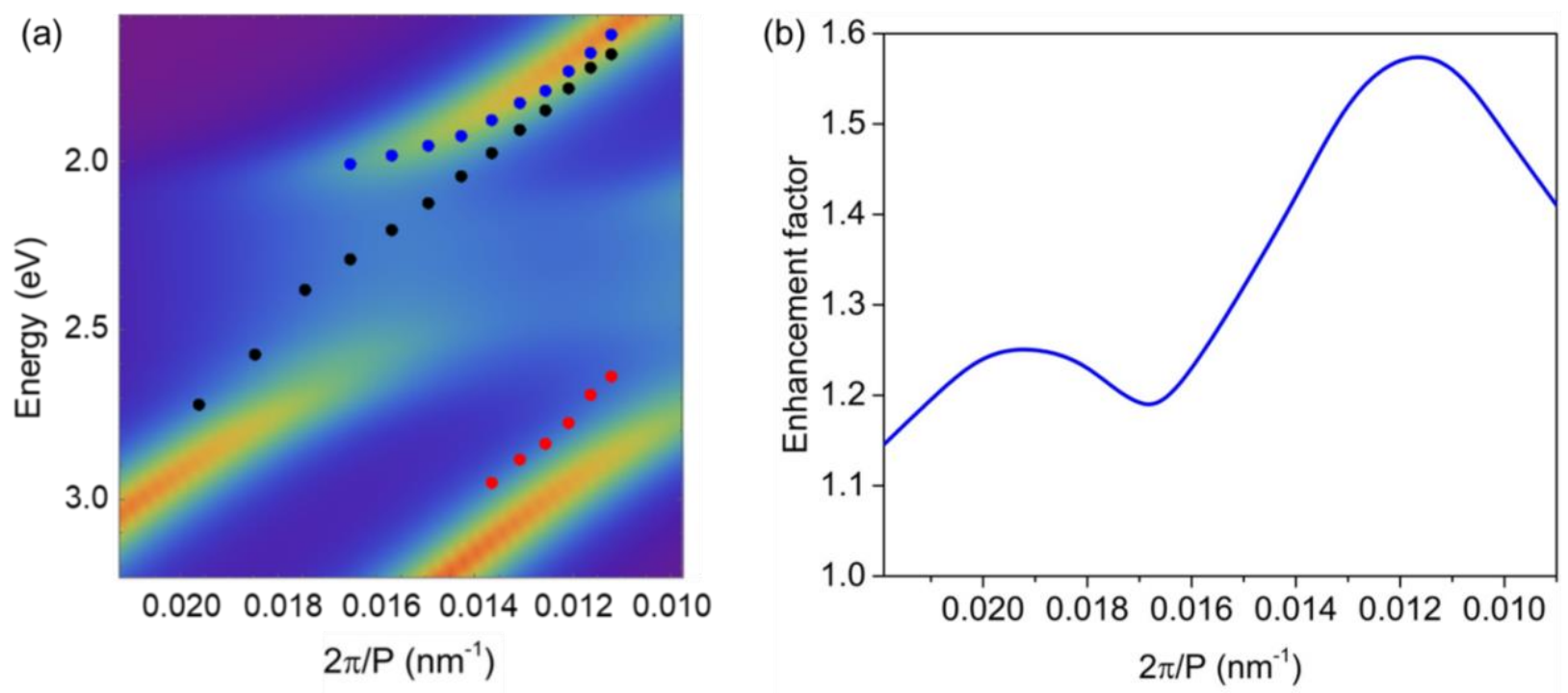

Figure 3. Results of the theoretical modelling: (a) the contour plot depicts the calculated photonic component of the polariton modes (cf. Supporting Information) as a function of frequency and $2 \pi / P$. Black and red points are experimental data for the bare $(1,0)$ and $(1,1)$ resonator modes respectively. The blue points are the experimentally measured polariton dispersion. (b) conductivity enhancement versus $2 \pi / \mathrm{P}$. In the calculation, the exciton transition energy is taken equal to $2.35 \mathrm{eV}$ with an inhomogeneous broadening equal to 0.3 $\mathrm{eV}$. The vacuum Rabi splitting is $0.6 \mathrm{eV}$. For the bare electronic scattering time, we have taken $\omega_{\text {exc }} \tau_{o}=0.1$ where $\hbar \omega_{\text {exc }}$ is the exciton transition energy.

considering a simplified model with two cavity modes and one disordered molecular ensemble with a Gaussian spread of the excitonic transition frequency. The value of the vacuum Rabi coupling and of the inhomogeneous broadening of the excitonic mode can be determined by fitting the cavity mode frequency dispersions, the available polariton dispersion and the exciton absorption spectrum, as depicted in Figure 3a. The only left adjustable parameter in the model is the Drude scattering time $\tau_{0}$ (associated to the mobility of the organic semiconductor) that can be determined by comparison to the experimental conductivity curves.47

The value of the scattering time that best fits the experimental behavior is consistent with the typical relatively-low mobilities of the considered organic semiconductors. In the $2 \pi / \mathrm{P}$ range of this study, this rather simple model predicts, as shown in Figure 3b, a conductivity enhancement, with two asymmetric peaks whose relative intensities and positions are rather close to the experimental values in Figure 2c. For the considered parameters, the theoretical peaks are broader than in the experiments and the maximum enhancement is around 1.6 instead of the measured value around 3. This difference can be ascribed to several factors, such as the actual 
disorder configuration of the experimental sample, which would require a much more sophisticated theoretical modeling, which is beyond the scope of this work.

We now turn to the photoconductivity measurements. Interestingly, the photoconductivity of rr-P3HT benefits from the enhanced conductivity (Figure S4, SI) under strong coupling in the same way as the dark current does, although the absolute values are higher under illumination (Figure 4). The photoconductivity also peaks for the resonant hole array $\left(\mathrm{P}=500 \mathrm{~nm} ; k_{\mathrm{x}}=0.0126\right.$ $\mathrm{nm}-1$ ) with an enhancement factor of $\sim 3$, when compared to that of on a random hole array (Figure S4, SI). Figure 4a shows the linear dependence of the photocurrent on the excitation intensity for rr-P3HT when measured under white light cw (continuous wave) illumination. At such low intensities per unit area $(<15 \mathrm{~mW}$ per $\mathrm{cm} 2)$, the fraction of excited state absorbers remains at all time negligible and as a consequence there is no significant depopulation of the ground state and the collective Rabi splitting is unmodified. The quantum yield for the photogeneration of charge carriers is estimated to be ca. 5 x 10-3 for rr-P3HT (520 nm illumination). The spectral response of the photocurrent was also studied using a tunable pulsed laser source (NKT laser) under strong coupling conditions and compared to that of a random hole array film. The ratio of the current for these two conditions is shown in Figure 4c and reveals enhanced photocurrent at longer wavelengths than the absorbance peak of the bare rrP3HT with a maximum around $620 \mathrm{~nm}$. This corresponds to the $P$ - peak. Since the spectral enhancement at long wavelengths is due to the Rabi splitting, a similar enhancement is expected to be seen at shorter wavelength corresponding to the $P+$ transition but our tunable laser system cannot reach the corresponding wavelengths.

For purposes of comparison, we also studied the photoconductivity of a perylene di-imide (PDI) n-type semiconductor under strong coupling (Figure S5, SI). We have already shown that this class of compounds exhibits an enhanced conductivity under strong coupling.11 The compound used here is a slightly different derivative whose structure is shown in the Supporting Information. Figure S5 (SI) summarizes the results. We observe that the photoconductivity of PDI is also enhanced upon ultra-strong coupling (Rabi splitting is $34 \%$ of transition energy, see Supporting Information) since two current peaks appear at the crossing of the PDI film absorbance peak and the plasmonic modes. Figure 4 a shows the linear dependence of the current with illumination power and Figure 4d shows that just as for rr-P3HT, the spectral response of PDI is enhanced where $P$-absorbs. 
(a)
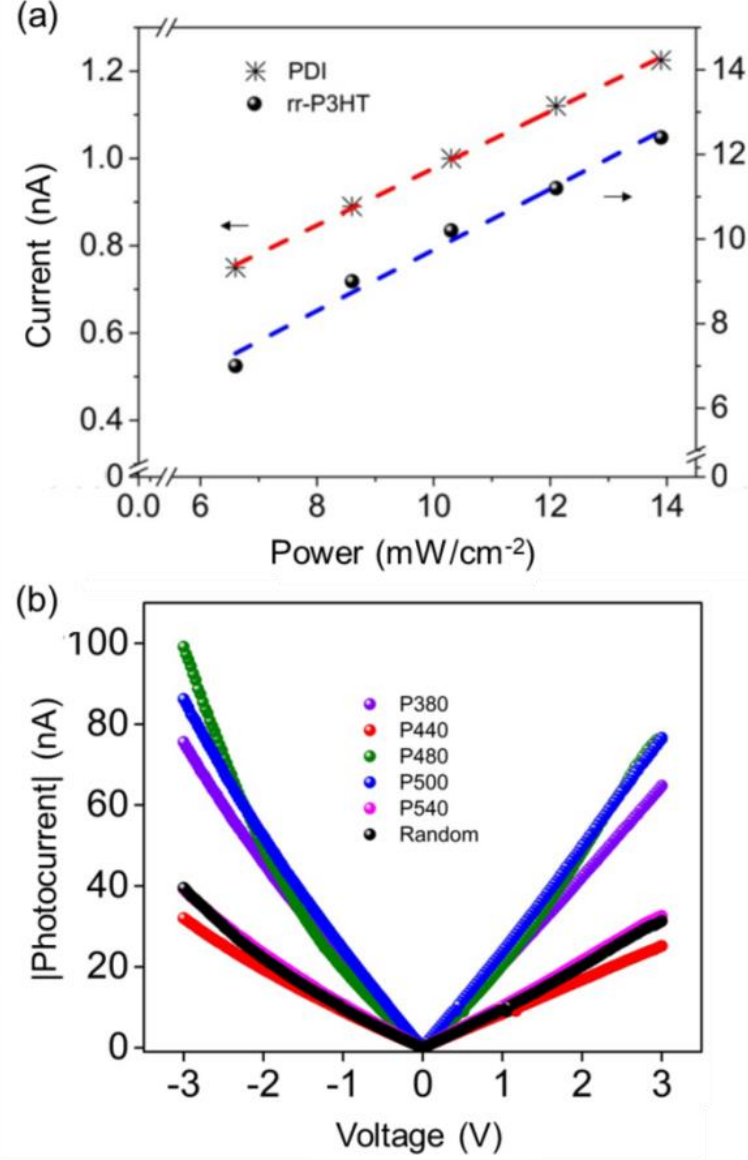

(c)

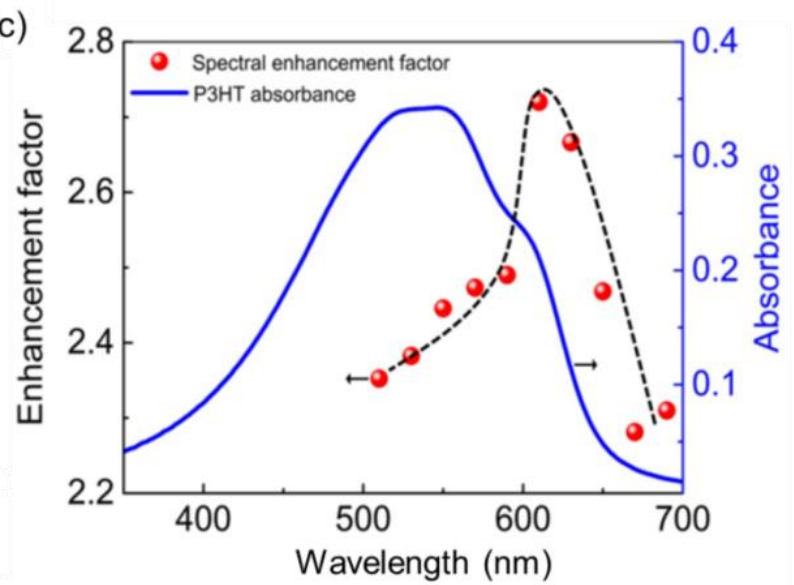

(d)

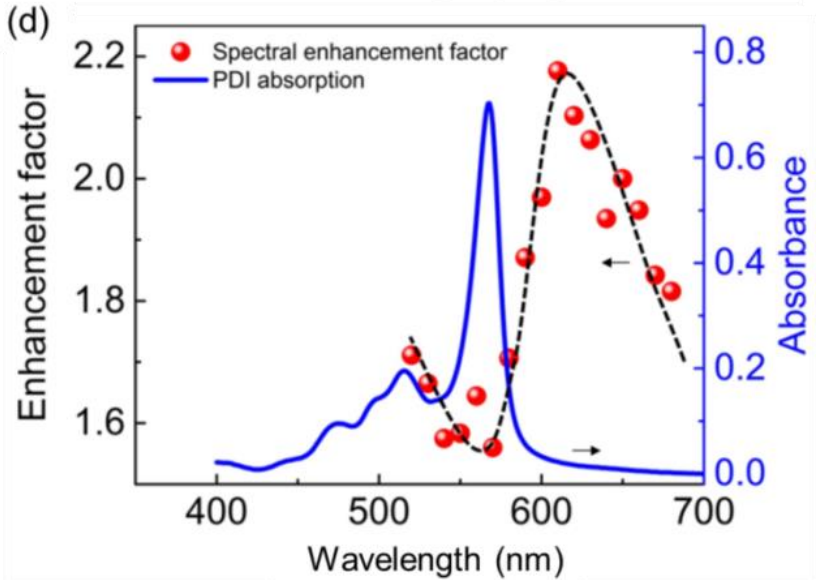

Figure 4. Photoconductivity under ultra-strong coupling: (a) Photocurrent as function of the white light illumination power for $\mathrm{rr}-\mathrm{P3HT}$ and $\mathrm{PDI}$ under strong coupling $(\mathrm{P}=500 \mathrm{~nm}$ and $P=280 \mathrm{~nm}$ respectively). (b) Current-Voltage (I-V) curves of rr-P3HT samples as a function of the period of the hexagonal hole array under white light illumination $(47 \mathrm{~mW}$ cm-2). (c) Spectral response under ultra-strong coupling (red dots, $P=500 \mathrm{~nm}$ ) as a function of excitation wavelength, the enhancement factor is the ratio of the photocurrent divided by the corresponding photocurrent of an uncoupled (random array) rr-P3HT film. A tunable pulsed laser with a $10 \mathrm{~nm}$ bandpass was used for this study. The peak at $620 \mathrm{~nm}$ corresponds to the $P$ - peak. The blue curve gives the absorbance spectrum of the bare rrP3HT film. The black lines are guides to the eye. (d) Idem for PDI under strong coupling $(P=280 \mathrm{~nm})$, the peak at $610 \mathrm{~nm}$ corresponds to $P$ - peak.

In conclusion, using a p-type organic semiconductor, we have demonstrated for the first time that hole transport in the ground state can also be enhanced by strong coupling. This is in turn a direct evidence of the polaritonic character of the valence band. We have also shown that the photoconductivity, like conductivity, can be boosted by the formation of the delocalized polaritonic states and in addition their spectral response can be extended and tailored by the coupling strength. This works for both $n$ - and p-type semiconductors. This proof-of-principle 
study opens the door for more detailed analysis of photoconductivity by time resolved pumpprobe techniques which will provide insight into the dynamics of charge carriers in the strong and ultra-strong coupling regimes. Strong coupling is easy to implement in a variety of configurations. We therefore expect that such results will find applications in devices where photoconductivity plays a role, in particular in photovoltaics and photodetectors. More broadly speaking, taken together with other investigations, it shows the potential of engineering the electromagnetic vacuum environment for material science and devices.

\section{Experimental Section}

\section{Synthesis of materials:}

Regioregular poly(3-hexylthiophene), rr-P3HT was synthesized and purified as reported earlier.46

\section{Two-terminal device with nanohole arrays for photoconductivity measurements:}

All the 2-terminal electrodes were realized on a glass substrate BK7 $(25 \times 25 \mathrm{~mm})$ after standard cleaning procedures with Hellmanex ${ }^{\mathrm{TM}}$ III solution (1.0\% solution in milliQ water) in a $35 \mathrm{kHz}$ sonication bath, then rinsed with water and sonicated for $1 \mathrm{~h}$ in (spectroscopically pure) ethanol. The glass substrates were then dried in an oven covered with Aluminium foil. $100 \mathrm{~nm}$ thick $\mathrm{Ag}$ electrodes were fabricated using a metallic cross mask containing 12 fingers (approx. $50 \mu \mathrm{m}$ width) in an electron beam evaporator (Plassys ME 300) at optimized working pressure ( 10-6 mbar) and deposition rates ( $\sim 2 \mathrm{~nm} \mathrm{~s}-1)$. Plasmonic hole arrays were generated by the NPVE software program and milled using a Carl Zeiss Auriga FIB system. $1.0 \mathrm{wt} \%$ of rr-P3HT and PDI solutions were freshly prepared by dissolving the molecules in spectroscopic grade trichloroethylene (Soxhlet extraction) and anhydrous chloroform respectively at ambient conditions and spin-coating onto the electrodes at 1000 r.p.m., to achieve $85 \mathrm{~nm}$-thick films. The reddish, smooth rr-P3HT and PDI thin-films were completely dried and annealed on a hot plate above the glass transition temperature. A passive layer of PVA was spin-coated ( $200 \mathrm{~nm})$ further to protect the active layer to avoid direct exposure to air and moisture. For a test experiment, in order to introduce an insulating layer in between the metal hole array and organic semiconductor, $20 \mathrm{~nm} \mathrm{SiO}_{\mathrm{x}}$ was sputtered on top of the metal hole array by using magnetron sputter under reduced pressure (10 mTorr) at $200 \mathrm{~W}$ for $50 \mathrm{sec}$. On top of the sputtered $\mathrm{SiO}_{\mathrm{x}}$, organic semiconductor and PVA layer were spin coated as explained above. 
The electrical characterization of the 2-terminal device was carried out by means of a Cascade Microtech MPS-150 probe station equipped with micro-positioners to contact the electrode pads. Both the dark current and photoconductivity I-V characteristics are recorded by means of a Keithley 2636B source meter interfaced with Labtracer 2.0 software. Photocurrent generation was tested under white light $\mathrm{cw}$ illumination and with a super-continuum ps pulsed laser (NKT Photonics) with a nominal power of $100 \mathrm{~mW}$ to $200 \mathrm{~mW}$, with variable wavelength (500-800 $\mathrm{nm} ; 10 \mathrm{~nm}$ FWHM) provided by a SuperK tunable single line filter. The output of the FC/PC fiber was directed to the center of the electrode covering a spot size of $2 \mathrm{~mm}$ in diameter.

\section{ASSOCIATED CONTENT}

Supporting Information contains Figures S1 to S5 and theoretical calculation details. The Supporting Information is available free of charge at https://pubs.acs.org.

\section{AUTHOR INFORMATION}

Corresponding Author

*E-mail: ebbesen@unistra.fr

Present Addresses

\$Present address: Department of Chemical Sciences, Indian Institute of Science Education and Research Mohali, Punjab, 140306, India.

\section{Author Contributions}

The manuscript was written through contributions of all authors. All authors have given approval to the final version of the manuscript.

\section{Funding Sources}

We acknowledge support of the International Center for Frontier Research in Chemistry (icFRC, Strasbourg), the ANR Equipex Union (ANR-10-EQPX-52-01), CSC (ANR-10-LABX- 0026 CSC) within the Investissement d'Avenir program ANR-10-IDEX-0002-02. TWE acknowledges the support of the ERC (project no 788482 MOLUSC). 
Notes

The authors declare no competing financial interest.

\section{REFERENCES}

(1) Ebbesen, T. W. Hybrid Light-Matter States in a Molecular and Material Science Perspective. Acc. Chem. Res. 2016, 49, 2403-2412.

(2) Törmä, P.; Barnes, W. L. Strong Coupling between Surface Plasmon Polaritons and Emitters: A Review. Rep. on Prog. Phys. 2014, 78, 013901.

(3) Kéna-Cohen, S.; Forrest, S. R. Room-Temperature Polariton Lasing in an Organic Single-Crystal Microcavity. Nat. Photonics 2010, 4, 371-375.

(4) Ballarini, D.; De Giorgi, M.; Cancellieri, E.; Houdré, R.; Giacobino, E.; Cingolani, R.; Bramati, A.; Gigli, G.; Sanvitto, D. All-Optical Polariton Transistor. Nat. Commun. 2013, 4, 1778.

(5) Plumhof, J. D.; Stöferle, T.; Mai, L.; Scherf, U.; Mahrt, R. F. Room-Temperature Bose-Einstein Condensation of Cavity Exciton-Polaritons in a Polymer. Nat. Mater. 2014, 13, 247-252.

(6) Chervy, T.; Xu, J.; Duan, Y.; Wang, C.; Mager, L.; Frerejean, M.; Münninghoff, J. A. W.; Tinnemans, P.; Hutchison, J. A.; Genet, C.; et al. High-Efficiency Second-Harmonic Generation from Hybrid Light-Matter States. Nano Lett. 2016, 16, 7352-7356.

(7) Aberra Guebrou, S.; Symonds, C.; Homeyer, E.; Plenet, J. C.; Gartstein, Yu. N.; Agranovich, V. M.; Bellessa, J. Coherent Emission from a Disordered Organic Semiconductor Induced by Strong Coupling with Surface Plasmons. Phys. Rev. Lett. 2012, 108, 066401.

(8) Shi, L.; Hakala, T. K.; Rekola, H. T.; Martikainen, J.-P.; Moerland, R. J.; Törmä, P. Spatial Coherence Properties of Organic Molecules Coupled to Plasmonic Surface Lattice Resonances in the Weak and Strong Coupling Regimes. Phys. Rev. Lett. 2014, 112, 153002.

(9) Hutchison, J. A.; Liscio, A.; Schwartz, T.; Canaguier-Durand, A.; Genet, C.; Palermo, V.; Samorì, P.; Ebbesen, T. W. Tuning the Work-Function Via Strong Coupling. Adv. Mater. 2013, 25, 24812485 .

(10) Thomas, A.; Lethuillier-Karl, L.; Nagarajan, K.; Vergauwe, R. M. A.; George, J.; Chervy, T.; Shalabney, A.; Devaux, E.; Genet, C.; Moran, J.; et al. Tilting a Ground-State Reactivity Landscape by Vibrational Strong Coupling. Science 2019, 363, 615.

(11) Orgiu, E.; George, J.; Hutchison, J. A.; Devaux, E.; Dayen, J. F.; Doudin, B.; Stellacci, F.; Genet, C.; Schachenmayer, J.; Genes, C.; et al. Conductivity in Organic Semiconductors Hybridized with the Vacuum Field. Nat. Mater. 2015, 14, 1123-1129.

(12) Hagenmüller, D.; Schachenmayer, J.; Schütz, S.; Genes, C.; Pupillo, G. Cavity-Enhanced Transport of Charge. Phys. Rev. Lett. 2017, 119, 223601.

(13) Feist, J.; Garcia-Vidal, F. J. Extraordinary Exciton Conductance Induced by Strong Coupling. Phys. Rev. Lett. 2015, 114, 196402.

(14) Schachenmayer, J.; Genes, C.; Tignone, E.; Pupillo, G. Cavity-Enhanced Transport of Excitons. Phys. Rev. Lett. 2015, 114, 196403. 
(15) Coles, D. M.; Somaschi, N.; Michetti, P.; Clark, C.; Lagoudakis, P. G.; Savvidis, P. G.; Lidzey, D. G. Polariton-Mediated Energy Transfer between Organic Dyes in a Strongly Coupled Optical Microcavity. Nat. Mater. 2014, 13, 712-719.

(16) Gonzalez-Ballestero, C.; Feist, J.; Moreno, E.; Garcia-Vidal, F. J. Harvesting Excitons through Plasmonic Strong Coupling. Phys. Rev. B 2015, 92, 121402.

(17) Zhong, X.; Chervy, T.; Zhang, L.; Thomas, A.; George, J.; Genet, C.; Hutchison, J. A.; Ebbesen, T. W. Energy Transfer between Spatially Separated Entangled Molecules. Angew. Chem. Int. Ed. 2017, 56, 9034-9038.

(18) Akulov, K.; Bochman, D.; Golombek, A.; Schwartz, T. Long-Distance Resonant Energy Transfer Mediated by Hybrid Plasmonic-Photonic Modes. J. Phys. Chem. C 2018, 122, 15853-15860.

(19) Vasa, P.; Wang, W.; Pomraenke, R.; Lammers, M.; Maiuri, M.; Manzoni, C.; Cerullo, G.; Lienau, C. Real-Time Observation of Ultrafast Rabi Oscillations between Excitons and Plasmons in Metal Nanostructures with J-Aggregates. Nat. Photonics 2013, 7, 128-132.

(20) Munkhbat, B.; Baranov, D. G.; Bisht, A.; Hoque, M. A.; Karpiak, B.; Dash, S. P.; Shegai, T. Electrical Control of Hybrid Monolayer Tungsten Disulfide-Plasmonic Nanoantenna Light-Matter States at Cryogenic and Room Temperatures. ACS Nano 2020, 14, 1196-1206.

(21) Li, J.; Ueno, K.; Uehara, H.; Guo, J.; Oshikiri, T.; Misawa, H. Dual Strong Couplings Between TPPS J-Aggregates and Aluminum Plasmonic States. J. Phys. Chem. Lett. 2016, 7, 2786-2791.

(22) Thomas, R.; Thomas, A.; Pullanchery, S.; Joseph, L.; Somasundaran, S. M.; Swathi, R. S.; Gray, S. K.; Thomas, K. G. Plexcitons: The Role of Oscillator Strengths and Spectral Widths in Determining Strong Coupling. ACS Nano 2018, 12, 402-415.

(23) Berrier, A.; Cools, R.; Arnold, C.; Offermans, P.; Crego-Calama, M.; Brongersma, S. H.; GómezRivas, J. Active Control of the Strong Coupling Regime between Porphyrin Excitons and Surface Plasmon Polaritons. ACS Nano 2011, 5, 6226-6232.

(24) Herrera, F.; Spano, F. C. Cavity-Controlled Chemistry in Molecular Ensembles. Phys. Rev. Lett. 2016, 116, 238301.

(25) Pellegrini, C.; Flick, J.; Tokatly, I. V.; Appel, H.; Rubio, A. Optimized Effective Potential for Quantum Electrodynamical Time-Dependent Density Functional Theory. Phys. Rev. Lett. 2015, $115,093001$.

(26) Rozenman, G. G.; Akulov, K.; Golombek, A.; Schwartz, T. Long-Range Transport of Organic Exciton-Polaritons Revealed by Ultrafast Microscopy. ACS Photonics 2018, 5, 105-110.

(27) Luk, H. L.; Feist, J.; Toppari, J. J.; Groenhof, G. Multiscale Molecular Dynamics Simulations of Polaritonic Chemistry. J. Chem. Theory Comput. 2017, 13, 4324-4335.

(28) Cirio, M.; De Liberato, S.; Lambert, N.; Nori, F. Ground State Electroluminescence. Phys. Rev. Lett. 2016, 116, 113601.

(29) Stranius, K.; Hertzog, M.; Börjesson, K. Selective Manipulation of Electronically Excited States through Strong Light-Matter Interactions. Nat. Commun. 2018, 9, 2273.

(30) Bartolo, N.; Ciuti, C. Vacuum-Dressed Cavity Magnetotransport of a Two-Dimensional Electron Gas. Phys. Rev. B 2018, 98, 205301. 
(31) Paravicini-Bagliani, G. L.; Appugliese, F.; Richter, E.; Valmorra, F.; Keller, J.; Beck, M.; Bartolo, N.; Rössler, C.; Ihn, T.; Ensslin, K.; et al. Magneto-Transport Controlled by Landau Polariton States. Nat. Phys. 2019, 15, 186-190.

(32) Guardado, J. O.; Salleo, A. Structural Effects of Gating Poly(3-Hexylthiophene) through an Ionic Liquid. Adv. Funct. Mater. 2017, 27, 1701791.

(33) Yan, H.; Chen, Z.; Zheng, Y.; Newman, C.; Quinn, J. R.; Dötz, F.; Kastler, M.; Facchetti, A. A High-Mobility Electron-Transporting Polymer for Printed Transistors. Nature 2009, 457, 679-686.

(34) Forrest, S. R. The Path to Ubiquitous and Low-Cost Organic Electronic Appliances on Plastic. Nature 2004, 428, 911-918.

(35) Arias, A. C.; MacKenzie, J. D.; McCulloch, I.; Rivnay, J.; Salleo, A. Materials and Applications for Large Area Electronics: Solution-Based Approaches. Chem. Rev. 2010, 110, 3-24.

(36) Himmelberger, S.; Salleo, A. Engineering Semiconducting Polymers for Efficient Charge Transport. MRS Commun. 2015, 5, 383-395.

(37) Salleo, A. Something out of Nothing. Nat. Mater. 2015, 14, 1077-1078.

(38) Ciuti, C.; Bastard, G.; Carusotto, I. Quantum Vacuum Properties of the Intersubband Cavity Polariton Field. Phys. Rev. B 2005, 72, 115303.

(39) Wang, Y. Photoconductivity of Fullerene-Doped Polymers. Nature 1992, 356, 585-587.

(40) Yasutani, Y.; Saeki, A.; Fukumatsu, T.; Koizumi, Y.; Seki, S. Unprecedented High Local ChargeCarrier Mobility in P3HT Revealed by Direct and Alternating Current Methods. Chem. Lett. 2012, $42,19-21$.

(41) Najafov, H.; Lee, B.; Zhou, Q.; Feldman, L. C.; Podzorov, V. Observation of Long-Range Exciton Diffusion in Highly Ordered Organic Semiconductors. Nat. Mater. 2010, 9, 938-943.

(42) O’Brien, G. A.; Quinn, A. J.; Tanner, D. A.; Redmond, G. A Single Polymer Nanowire Photodetector. Adv. Mater. 2006, 18, 2379-2383.

(43) Irkhin, P.; Najafov, H.; Podzorov, V. Steady-State Photoconductivity and Multi-Particle Interactions in High-Mobility Organic Semiconductors. Sci. Rep. 2015, 5, 15323.

(44) Huang, Z.; Zhou, W.; Huang, J.; Wu, J.; Gao, Y.; Qu, Y.; Chu, J. Directly Tailoring PhotonElectron Coupling for Sensitive Photoconductance. Sci. Rep. 2016, 6, 22938.

(45) Walsh, J. J.; Lee, J. R.; Draper, E. R.; King, S. M.; Jäckel, F.; Zwijnenburg, M. A.; Adams, D. J.; Cowan, A. J. Controlling Visible Light Driven Photoconductivity in Self-Assembled Perylene Bisimide Structures. J. Phys. Chem. C 2016, 120, 18479-18486.

(46) Nawaz, A.; Meruvia, M. S.; Tarange, D. L.; Gopinathan, S. P.; Kumar, A.; Kumar, A.; Bhunia, H.; Pal, A. J.; Hümmelgen, I. A. High Mobility Organic Field-Effect Transistors Based on Defect-Free Regioregular Poly(3-Hexylthiophene-2,5-Diyl). Org. Electron. 2016, 38, 89-96.

(47) Naudet-Baulieu, C.; Bartolo, N.; Orso, G.; Ciuti, C., Dark Vertical Conductance of CavityEmbedded Semiconductor Heterostructures, New J. Phys. 2019, 21, 093061. 